\title{
Dental oncology in patients treated with radiation for head and neck cancer
}

\author{
Rex Hoffman, MD, James Kelly, DDS, MS, ${ }^{\mathrm{b}}$ Earl G Freymiller, DMD, MD, ${ }^{\mathrm{c}}$ Daniel P \\ Copps, DDS, ${ }^{d}$ and Ian V Hutchinson, PhD, DSc
}

\begin{abstract}
${ }^{a}$ Department of Radiation Oncology, Roy and Patricia Disney Family Cancer Center, St Joseph Medical Center, Burbank, California; ${ }^{b}$ Department of Dental Specialties, Mayo Clinic, Rochester, Minnesota; 'Division of Diagnostic and Surgical Services, Section of

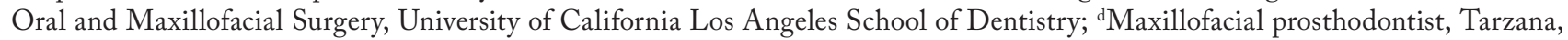
California; and ${ }^{\mathrm{e} P r o v i d e n c e ~ H e a l t h}$ and Services, Burbank, California
\end{abstract}

\begin{abstract}
The dentition of head and neck cancer patients is of utmost importance when they receive radiation therapy, especially because patients are living longer after a course of head and neck radiation. Good communication among the oncology team members (the radiation and medical oncologists, the maxillofacial prosthodontist/dental oncologist, otolaryngologist, reconstructive surgeon, nursing support) and the patient is essential initially, and subsequently including the general dentist as well. The maxillofacial prosthodontist/dental oncologist has a very important role to play before, during, and after radiation therapy, to avoid loss of supporting bone and teeth and damage to soft tissues. Each member of the oncology team should have a basic knowledge of the patient's dentition and supporting bony structure, including the numbering of teeth. There are treatment-related issues of concern for the dental oncologist, so the treating oncologist must provide proper information to ensure appropriate dental care. Post-radiation dental care may be assumed by a general dentist for long-term maintenance. The effectiveness of the education of caregivers and head and neck cancer patients in reducing osteoradionecrosis (ORN) has been demonstrated. As the number of patients developing human papilloma virus-related head and neck cancer continues to increase, greater emphasis must be placed on good dental care. The aim of this primer for all those caring for patients with head and neck cancer is to underscore the important role of the dental oncologist during all phases of radiation therapy, and to provide guidelines to minimize and prevent dental complications such as radiation-induced caries and ORN.
\end{abstract}

$\mathrm{T}$ o ensure that head and neck cancer patients achieve optimal results during and after receiving radiation therapy, either with or without chemotherapy or biologics, it is essential that there is good communication between different members of the oncology team. This team generally includes the radiation and medical oncologists, the maxillofacial prosthodontist/dental oncologist, otolaryngologist, reconstructive surgeon, nursing support, and the patient's general dentist. This primer is intended to provide information to the whole team of those caring for head and neck cancer patients so that, through collaboration, they can ensure their patients maintain good dentition, not only during their course of therapy but for years to come.

\section{Pre-treatment evaluation}

An evaluation by the dental oncologist is required as soon as the decision is made to use radiation therapy in a head and neck cancer patient. Both the radiation and medical oncologist should be conversant with the basic anatomy of the oral cavity and the num- bering of the permanent teeth. The universal dental numbering scheme refers to teeth sequentially from 1-32, starting with the maxillary right third molar and proceeding around the maxillary dental arch to the left third molar (Figure 1). The next tooth in order is the mandibular left third molar, and numbering continues around the mandibular arch to the right third molar. Communicating to the dental oncologist the structures included in the radiation field and their predicted dose of radiation is imperative to allow the dental oncologist to determine the risk factors for potential short- and long-term medical and dental problems. These may include radiation-induced caries and osteoradionecrosis (ORN), as well as possible damage to the gums and soft tissues, xerostomia, trismus, velopharyngeal inadequacy, mucositis, dysphagia, and dysgeusia.

These discussions should take place before a patient starts radiation. Such exchange of information can translate into fewer treatment-related side effects. It allows the radiation oncologist and the dental oncologist to describe to each other their den-

Accepted for publication August 29, 2016. Correspondence: lan V Hutchinson, PhD, DSc; ian.hutchinson@providence.org. Disclosures: The authors report no disclosures or conflicts of interest. JCSO 2016;14(9):374-379. (02016 Frontline Medical Communications. doi: 10.12788/jcso.0294. 


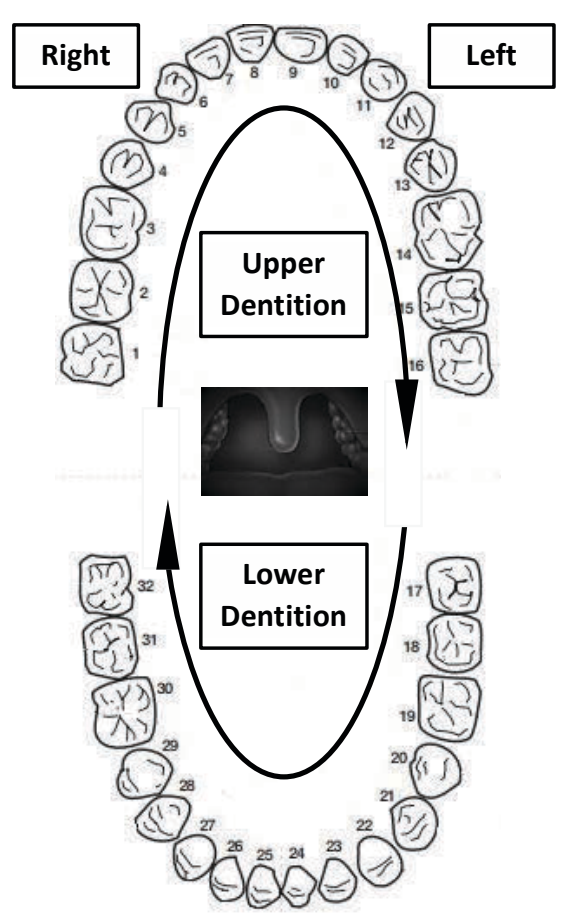

FIGURE 1 The universal numbering system of permanent teeth, as seen looking into the patient's mouth.

tal findings on both their clinical and radiographic exams. This may alert the dental oncologist and the oncology team to potential dental issues that should be addressed before starting a course of radiation therapy. It is essential, too, that other aspects of the patient's history and physical condition be evaluated before embarking on a course of radiation (Table 1).

As a result of this examination, the maxillofacial prosthodontist/dental oncologist or oral maxillofacial surgeon may remove teeth before radiation therapy, to reduce the risk of ORN. Teeth with a poor prognosis should be carefully removed, preferably 7-10 days before the initiation of radiotherapy, to allow sufficient healing of the primary closure and appropriate alveoloplasty.

Treatment planning for patients undergoing radiation is multifactorial and includes defining the fields of radiation, establishing dental status, and assessing patient compliance factors. A lack of patient motivation should lead to the extraction of any questionable teeth before radiation treatment. The deciphering of the extraction conundrum has to consider radiation exposure, type, field, and dosage. For example, when irradiating a lesion at the base of the tongue, teeth in the anterior mandible, anterior to the mental foramen, are generally at low risk of ORN because they will likely receive less than 50 Gray. By contrast, posterior mandibular teeth receive a cumulative dose of 68-72 Gray.

Tissue positioners or mouth guards can attenuate radiation damage (Figure 2). The optimal design and position-
TABLE 1 Synopsis of the different aspects of a patient's history, examination, and work-up for evaluating a head and neck cancer patient before starting a course of radiation

\section{History}

Past medical history

Past dental history

Current medications

Drug or material allergies

Social history (ie alcohol, tobacco, illicit drug use)

Examination

Extra-oral

Check cervical lymph nodes bilaterally

Assess temporomandibular joint

Check baseline maximal incisal opening

Intra-oral

Check for caries

Check for periodontal disease

Check for soft tissue pathology, including buccal mucosa, gingival mucosa, floor of mouth, tongue, palate, and tonsils

Assess salivary quality and quantity

\section{Radiographic examination}

Panoramic imaging

Selective periapical images of all teeth

Check for dental cysts

Check for periodontal disease

Check for gross tooth decay (caries)

Dental abscesses

External or internal root resorption

Ectopic eruption
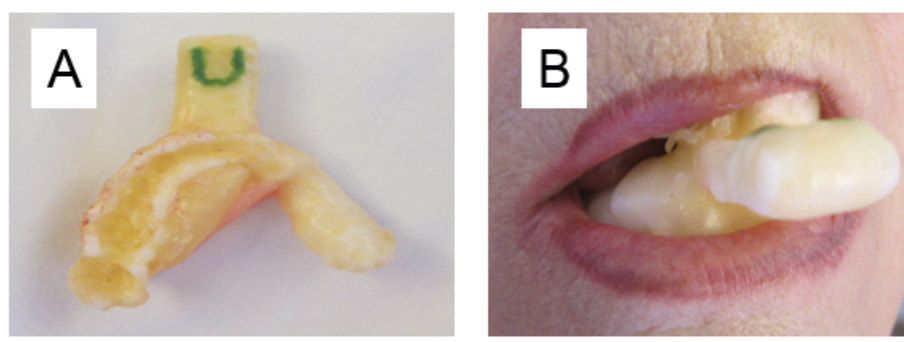

FIGURE 2 A tissue positioner designed for a patient being treated to the left parotid tumor bed. A, The tissue positioner as it was presented to the patient, with the $U(U p)$ when it was introduced into the mouth. When in place, the positioner pushed the tongue to the right side of the mouth, out of the beam of radiation, to decrease the possible radiation toxicity to the tongue, namely loss of taste and mucositis. B, The positioner in place, before application of the face mask. 
ing of a tissue positioner requires collaboration between the radiation oncologist and the dental oncologist before radiotherapy begins. Intra-oral prostheses can position oral tissues into or away from the beam of radiation. They also help to immobilize and stabilize the oral cavity structures such as the tongue, to ensure that they are in a repeatable position for each administration of radiation. For unilateral lesions in areas such as the parotid glands, buccal mucosa, tonsil, and retro-molar trigone, a positioner can hold the oral tongue to the unaffected side while keeping the maxilla and mandible in the same position during each treatment, in turn decreasing the comorbidities associated with radiation therapy (Figure 2).

Radiation spacing mouth guards are particularly useful for patients who have extensive metal or porcelain crowns and/or gold, porcelain, or silver fillings. The direct beam of radiation will pass through such mouth guard material, but will create a space between the restoration and the cheek or tongue. This decreases the back and forward scatter of radiation, thereby decreasing the magnitude of mucositis in these areas.

Before starting radiation therapy, custom-designed soft fluoride carriers are also made so that the patient can use topical fluorides before, during, and after therapy. ${ }^{1}$ Use of these fluoride carriers decreases the likelihood of a patient developing caries. Some people may develop heightened sensitivity in their teeth. Mouth guards can also be created to prevent biting of irradiated tissues that might become swollen during treatment.

Edentulous patients are also advised to practice good oral hygiene, and to avoid trauma and premature use of prostheses. Those undergoing surgery should be prepared for necessary prosthetic rehabilitation to correct surgical defects. This rehabilitation should not take place until after radiation treatment, and is totally dependent on the condition of the hard- and soft-tissue repair following radiation therapy and patient comfort.

It is essential that a patient is counselled on the importance of maintaining a healthy oral status before embarking on therapy to decrease the risk of developing major oral complications in the future. Patients should be advised to avoid alcohol use and smoking, and not to eat coarse, spicy, or hot foods that may traumatize the soft tissues. It is also important to emphasize avoidance of alcohol-based mouth rinses and/or phenol that can exacerbate mucositis. ${ }^{2}$ Flossing should be done carefully.

\section{Evaluation during treatment}

Properly evaluating and caring for teeth before starting a course of radiation greatly reduces the patient's risk of developing complications to the head and neck. Complications can be either acute (mucositis, infectious stomatitis, altered taste and smell, dermatitis, pain, inflammation, and difficulty swallowing) or chronic (xerostomia, dental caries, fibrosis, trismus, photosensitivity, ORN, and chronic nerve pain). The severity of these treatment-induced morbidities depends on several factors, including the dose of radiation, energy source, volume of tissue treated, pre-treatment performance status, and pre-existing periodontal disease.

With radiation to the head and neck region that includes one or both of the parotid glands, submandibular glands, and sublingual glands, there is a decrease in the production of saliva function (xerostomia) that reduces the natural lavage of food and microbial debris within the oral cavity, and can lead to the development of dental caries. Therefore, a salivary stimulant such as pilocarpine should be started 3 days before treatment to increase salivary function. ${ }^{3,4}$ This should be taken preferably with a fatty food to eliminate or greatly diminish flushing or sweating. Salivary stimulants should not be taken on an empty stomach. Contraindications to taking this medication include glaucoma, severe eye infection, eye inflammation, or uncontrolled asthma. Because of pilocarpine's cholinergic effects, it should not be taken with an anti-cholinergic drug such as atropine or with beta blockers such as atenolol.

With xerostomia, there are changes in the oral flora that reduce the $\mathrm{pH}$ level and buffering capacity, which can lead to a cariogenic oral environment. With a decrease in salivation, there can be a big increase in Streptococcus mutans, a bacterium causing tooth decay, and the yeast Candida albicans. ${ }^{5}$ It is important to review the patient's diet and discourage intake of sugar carbohydrates.

Maintaining good oral hygiene during radiation therapy is essential for improving oral comfort and greatly reduces oral lesions during treatment. Bacterial and fungal infections can occur. Oral rinsing with a solution of a teaspoon of sodium bicarbonate dissolved in a glass of water, several times a day, creates a buffering capacity and raises the $\mathrm{pH}$ level which are affected by xerostomia. This use of a sodium bicarbonate solution may assist in decreasing oral microorganisms and maintaining mucosal hydration. During treatment, one should eliminate secondary sources of irritation such as alcohol, smoking, citric acids, coarse foods, hot foods, and alcohol or phenol-containing mouth rinses.

During treatment it is essential to use fluoride gel daily to minimize radiation tooth caries. Patients should use fluoride carriers with a $0.4 \%$ stannous fluoride gel and place them onto the dentition for 5 minutes. Thereafter, no fluids or food should be consumed for 30 minutes. Patients should be monitored weekly during radiation therapy to ensure compliance with fluoride applications and oral hygiene if possible.

Good oral hygiene can greatly reduce oral complications during and after the completion of treatment.

\section{Post-treatment evaluation}

When radiation treatment has been completed, the radiation oncologist sends the dental oncologist a copy of the 
treatment summary and dose-volume histogram (DVH) and selected colored anatomical schematics that clearly show the dose levels to the structures that were treated (Figure 3). This allows the dental oncologist to clarify the risk factors for future dental procedures (both routine and complex). Head and neck cancer patients who have had extensive surgical procedures, such as maxillectomy or mandibulectomy, are usually restored and maintained by the maxillofacial prosthodontist/dental oncologist. In most cases, the dental oncologist will follow the patient on a limited basis for the first 2-3 months after treatment cessation, until the mucositis has cleared up and to assess salivary content. After that time, the patient should be referred back to the general dentist for close routine dental maintenance.

It is essential that each care provider in the oncology team, including the radiation oncologist and medical oncologist, re-emphasizes the use of daily fluoridation with the trays. Dental caries are a common sequel in head and neck cancer patients for 6-12 months after irradiation and can continue throughout life. Irradiation of the salivary glands decreases the quantity of saliva, putting patients at increased risk of caries if they ingest a diet high in carbohydrates and sucrose. ${ }^{6}$ When a patient's follow-up and oral hygiene are compromised, rampant dental caries will likely develop. Because
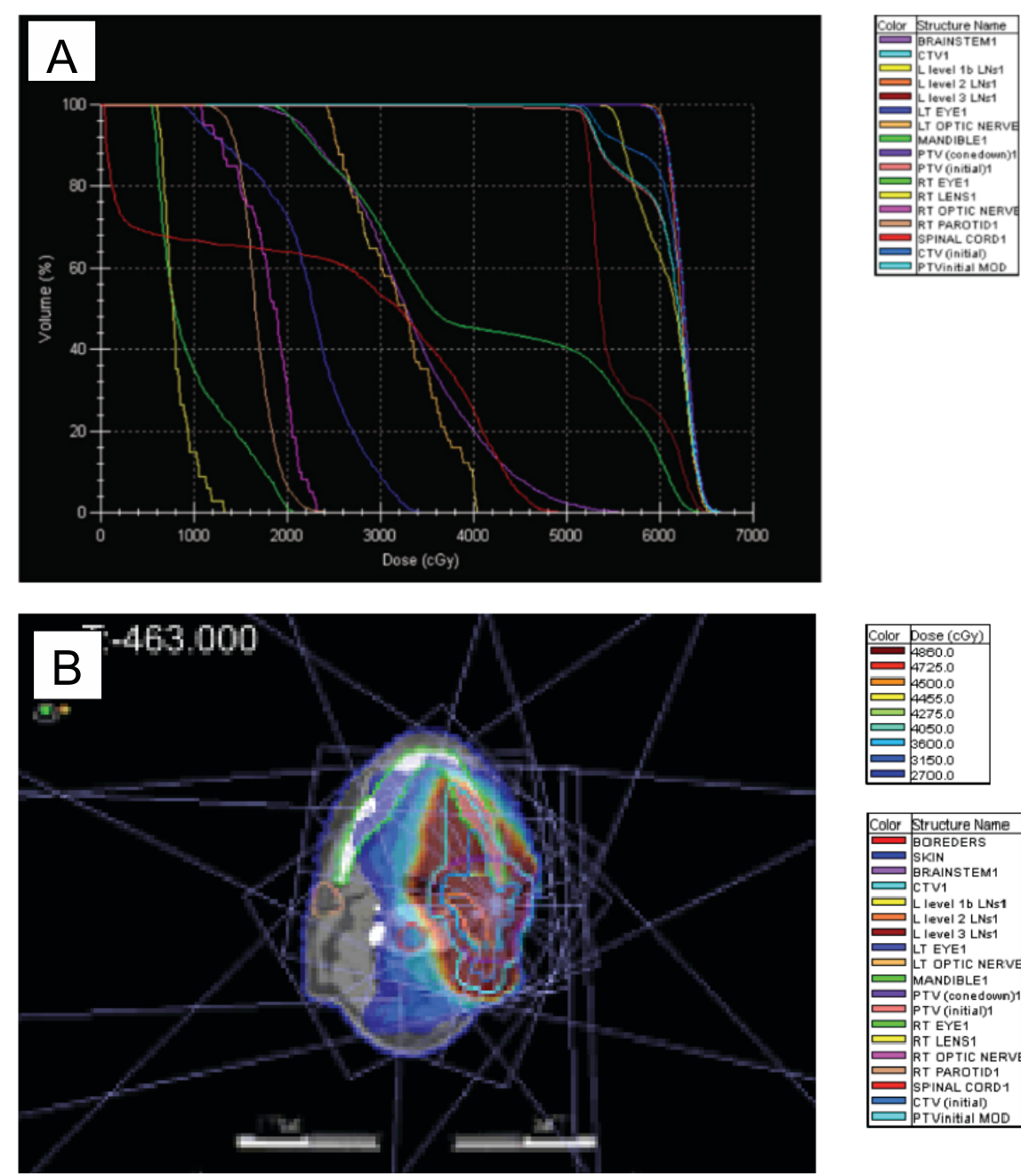

FIGURE 3 The dose of radiation to each oral structure can be determined from $\mathbf{A}$, the dose-volume histogram or $\mathbf{B}$, the colored anatomical schematic. of this, it is imperative that either a $0.4 \%$ stannous fluoride or $1.1 \%$ sodium fluoride tooth paste (depending on the saliva rebound) be continued indefinitely after the course of radiation..$^{6-8}$

It should be noted that saliva rebound can occur in many patients, which can greatly reduce xerostomia, and that a regular over-the-counter tooth paste will not prevent radiation-induced tooth decay. Patients should be maintained on their sialagogue therapy (such as pilocarpine) for 12 weeks after treatment cessation., ${ }^{3-11}$ We have found that pilocarpine used beyond that time frame has not been beneficial.

Radiation oncologists should always encourage head and neck cancer patients to contact them if their dentist indicates that they need a tooth extraction, "deep" scaling, periodontal surgery, a dental implant, or an orthodontics procedure - the risk of complications after trauma or oral surgical procedures in an irradiated field can be significant.

Therefore, elective surgical procedures such as an extraction, implant placement, or soft-tissue surgery are contraindicated within a previously highly irradiated field.

If oral surgery is not elective, it is critical that the dose of radiation delivered to the tissue beds be evaluated and identified through the use of the DVH, which illustrates the isodose curves through specific parts of the mandible. The treatment summary should be relayed to the dental or medical onclologist and/or the oral maxillofacial surgeon to determine the risk factors for ORN that could be caused by simple procedures such as a tooth extraction.

Prophylactic use of antibiotics should be continued until a surgical risk can be evaluated with the DVH and summary report. Having a tooth extracted from an area that has received more than 50 Gray puts a patient at increased risk for developing ORN. ${ }^{12}$ Pre-operative use of hyperbaric oxygen (HBO) therapy will generally increase the blood flow into the respective dental site and minimize the risk of ORN 


\section{How We Do It}
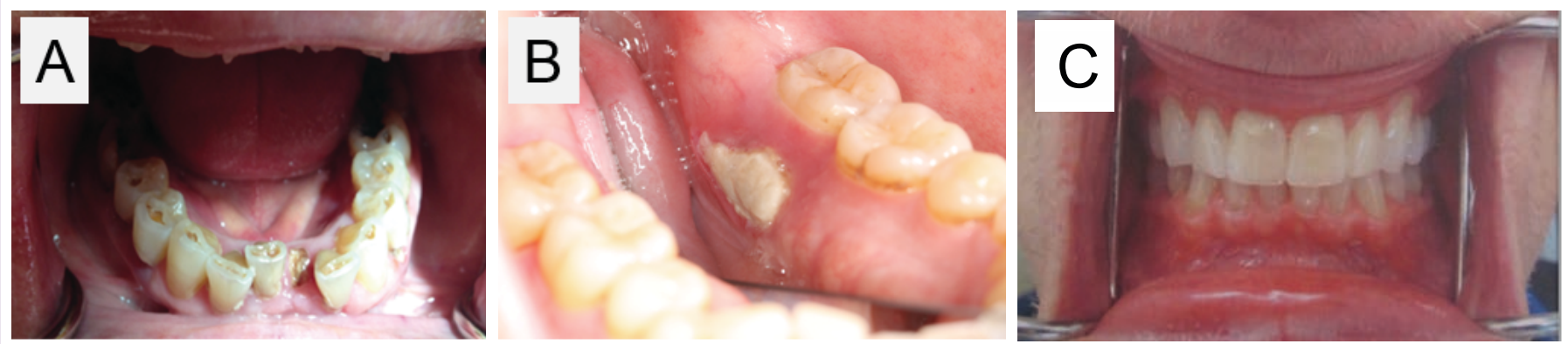

FIGURE 4 Dental caries (A) and osteoradionecrosis (B) occurring after cessation of radiation, and $\mathbf{C}$, perfect dentition in a patient 4 years after radiation for stage IVA tonsil cancer.

by promoting angiogenesis and osteogenesis. $\mathrm{HBO}$ must be used as an adjunct to debridement with primary non-tension tissue closure and followed with antibiotics for 10 days. ${ }^{13,14}$ Such therapy usually consists of 20 treatments, followed by surgery, then another 10 treatments. ${ }^{15}$ When patients are properly screened, hyperbaric oxygen treatment has been found to be safe for these individuals with irradiated tissues. ${ }^{16}$

Other non-surgical procedures such as oral prophylaxis, imaging, routine restorative procedures, endodontics, and prosthodontics can be performed safely on a routine basis without special treatment. Sometimes it is necessary to premedicate these patients with antibiotics and use hyperbaric oxygen treatment. The dental structures, both hard and soft tissues, which receive more than 50 Gray of radiation are at high risk for an ORN if an invasive dental surgical procedure is performed.

TABLE 2 Guidelines for post-radiation oral care in patients with head and neck cancers

The patient should apply fluoride daily, using an applicator. Fluoride mouth washes are inadequate to prevent caries.

Continue routine dental maintenance, including scaling $2 \mathrm{~mm}$ below the gingival crest. Exceptions are:

- Do not perform gingival curettage or deep

cleaning in the field of primary radiation.

- Do not use electronic cavitron scalers to remove calculus.

If restorative dentistry is necessary, the procedure must be done with little or no hemorrhage of the soft tissues.

Extractions and peridontal surgery put the patient at very high risk of osteoradionecrosis. Such procedures must not be performed without consultation with the dental and radiation oncologists.

The patient should be reviewed for the following: - Incisal tooth caries

- Gross cervical caries ('root caries')

- Candida albicans fungal infection

- Black tongue

- Open boney exposures or sores

- Any other issues appearing post radiation
Osteoradionecrosis has been defined in different ways by different authors. We define it as "the presence of nonvital bone in a proven radiation oncology field." The risk of ORN depends on the size and location of the lesions, volume of mandible in the radiation field, dose of radiation, and the health of the patient's dentition. When a primary tumor is adjacent to or overlies bone, the risk of ORN significantly increases.

If an ORN appears within the first 12 months after a course of radiation therapy, treatment should be conservative. In most cases, these can be cared for by local irrigation, minimal debridement and a periodic systemic antibiotic. Good oral hygiene is paramount. Over time, the osseus fragment will exfoliate and heal over. It is imperative that the clinician have patience, because bony exposure in an irradiated bed often requires an extended time to heal.

One conservative approach some clinicians have taken when treating a patient with ORN of the jaw is the combined use of pentoxifylline and vitamin $\mathrm{E}$ which is aimed at reducing fibrosis. ${ }^{17,18}$ Although results from such treatment seems good in some cases, a phase 3 randomized trial is needed.

Most cases of an ORN occur in the posterior mandible, although cases have been reported in the maxilla, too. Extraction of maxillary teeth in the radiated field may be performed after radiation therapy with little risk of bony infection. The extraction must be done carefully and be as atraumatic as possible. The sutures should be left in place for a couple of weeks to allow slow healing to take place. The patient should be covered with prophylactic antibiotics during this phase of healing.

Our policy has been for the dental oncologist to send a follow-up letter back to the patient's general dentist that spells out procedures that can and, especially, cannot be done for these patients. Without following the recommended guidelines for post-radiation care (Table 2), patients who have received radiation therapy to the head and neck are at increased risk for the development of both dental caries and ORN (Figure 4). Alternatively, with proper dental care before, during, and after a course of radiotherapy, the 
patients' teeth can be maintained in great shape (Figure 4).

A knowledge and understanding of the dental needs of head and neck cancer patients can reduce the incidence of dental and medical complications following a course of radiation. This is a responsibility shared by the radiation oncologist, the medical oncologist, the dental oncologist, the patient's dental care provider, and the rest of the oncology team, so good communication between members of this team is essential.

\section{Conclusion}

This primer outlines the "way we do it" in terms of dental

\section{References}

1. Fleming TJ. Oral tissue changes of radiation-oncology and their management. Dent Clin North Am. 1990;34:223-237.

2. Borowski B, Benhamou E, Pico JL, et al. Prevention of oral mucositis in patients treated with high-dose chemotherapy and bone marrow transplantation: a randomized controlled trial comparing 2 protocols of dental care. Eur J Cancer B Oral Oncol. 1994;30B:93-97.

3. Johnson JT, Feretti GA, Nethers WJ, et al. Oral pilocarpine for postirradiation xerostomia in patients with head and neck cancer. N Engl J Med. 1993;329:390-395.

4. Le Veque, FG, Montgomery M, Potter D, et al. A multicenter, randomized double-blind, placebo controlled, dose-titration study of oral pilocarpine for treatment of radiation-induced xerostomia in head and neck cancer patients. J Clin Oncol. 1993;11:1124-1131.

5. Chen TY, Webster JH. Oral monilia study on patients with head and neck cancer during radiotherapy. Cancer. 1974;34:246-249.

6. Keene HJ, Fleming. Prevalence of caries-associated microflora after radiotherapy in patients with cancer of the head and neck. Oral Surg Oral Med Oral Pathol. 1987;64:421-426.

7. Chambers MS, Toth BB, Martin JW, et al. Oral and dental management of the cancer patient: prevention and treatment of complications. Support Care Center. 1995;3:168-175.

8. Scarantino C, LeVeque F, Swann RS, et al. Effect of pilocarpine during radiation therapy : results of RTOG 97-09, a phase III randomized study in head and neck cancer patients. J Support Oncol. 2006;4(5):252-258.

9. Zyarady Z, Nemeth A, Ban A, et al. A randomized study to assess the effectiveness of orally administered pilocarpine during and after radiotherapy of head and neck cancer. Anticancer Res. 2006; 26 (B):1557-1562.

10. Lovelace TL, Fox NF, Sood AJ, et al. Management of radiother- care before radiation for head and neck cancer and after treatment. The authors hope is that this presentation provides an overview of the dental complications of head and neck radiation, and results in future head and neck cancer patients being less likely to develop unnecessary dental/medical complications following a course of radiation therapy.

\section{Acknowledgments}

The authors acknowledge Nancy Ellerbroek, MD, FACR, a radiation oncologist at Valley Radiotherapy Associates in Los Angeles, California, for her input and guidance in creating this primer.

apy-induced salivary hypofunction and consequent xerostomia in patients with oral or head and neck cancer: meta-analysis and literature review. Oral Surg Oral Med Oral Pathol Oral Radiol. 2014;117(5):595-607.

11. Zimmerman RP, Mark RJ, Tran LM, et al. Concomittant pilocarpine during head and neck irradiation is associated with decreased post-treatment xerostomia. Int J Radiat Oncol Biol Phys. 1997:37(3):571-575.

12. Morrish RB Jr, Chan E, Silverman S Jr, et al. Osteonecrosis in patients irradiated for head and neck carcinoma. Cancer. 1981;47:1980-1983.

13. Marx RE, Johnson RP. Studies in the radiobiology of ORN and their clinical significance. Oral Surg Oral Med Oral Pathol. 1987;64:379-390.

14. Mansfield MJ, Sanders DW, Heimbach RD. Hyperbaric oxygen as an adjunct in the treatment of ORN of the mandible. J Oral Surg. 1981;39:585-589.

15. Marx RE, Johnson RP, Kline SN. Prevention of ORN: a randomized prospective clinical trial of hyperbaric oxygen versus penicillin. J Am Dent Assoc. 1985;111:49-54.

16. Grimm PS, Gottlieb LJ, Boddie A, et al. Hyperbaric oxygen therapy. JAMA. 1990;263:2216-2220.

17. Delanian S, Chatel C, Porcher R, et al. Complete restoration of refractory mandibular ORN by prolonged treatment with a pentoxyifylline- tocopherol-clodronate combination (PENTOCLO): a phase II trial. Int J Radiat Oncol Biol Phys. 2011;80(3):832-839.

18. Kahenasa N, Sung EC, Nabili V, et al. Resolution of pain and complete healing of mandibular ORN using pentoxifylline and tocopherol. Oral Surg Oral Med Oral Pathol Oral Radiol. 2012;113(4):e18-23. 